\title{
Antibody induction by influenza vaccines in the elderly: a review of the literature
}

\author{
Walter E.P. Beyer*, Abraham M. Palache ${ }^{\dagger}$, Machteld Baljet ${ }^{\dagger}$ and Nic Masurel*
}

\begin{abstract}
Conflicting results have been reported concerning the association between high age and response to influenza vaccines. Some authors have found a reduced response in aged subjects, others have found no difference or even better results as compared with younger control subjects. Seventeen papers were selected from international literature published in the period 1968-1988 for a review of the anti-haemagglutinin-IgG sero-response following vaccination: among 30 cases in which vaccine components could be studied independently, ten revealed a better immune response in young subjects than in the elderly, four found more favourable results in the elderly, and 16 could not detect any significant between-group-differences, the latter most probably because of a high type-2-error. Nine of these 16 cases tended to favour young subjects. These results were relativated by the finding that each paper had at least one of three methodological limitations: (1) the failure to exclude subjects with illnesses or using drugs influencing the immune system, (2) the failure to exclude subjects with previous vaccinations against influenza, (3) the failure to exclude subjects with high prevaccination antibody titres. The direction of these biases is such that failure to address any one issue will lead to an underestimate of the response of aged subjects. In view of the failure to control these biases, it was not surprising that the papers reviewed presented a heterogeneous picture. Thus, the association between high age per se and response to influenza vaccines, if any, has not yet been established. Suggestions are made for future studies in which admission criteria should control health state and previous exposure to influenza antigens.
\end{abstract}

\section{Introduction}

The proportion of people older than 60 years within the total population of developed countries is rapidly growing; the control of disorders related to higher age will increasingly become a focus of the entire health system. Human influenza viruses, naturally occurring during pandemics (influenza $\mathrm{A}$ ) and epidemics (influenza $A$ and $B$ ) cause significant excess morbidity and mortality in the elderly ${ }^{1,2}$, not only in those with underlying

*Department of Virology and WHO Influenza Centre, Erasmus University, PO Box 1738, 3000 DR Rotterdam, The Netherlands. 'Duphar BV, Department of Clinical Research, Weesp, The Netherlands. (Received 5 October 1988; revised 26 April 1989; accepted 10 May 1989) age-related chronic diseases, but also in apparently healthy subjects ${ }^{3,4}$. Thus, prevention of influenza virus infections in the aged would substantially contribute to longevity and state of health. However, active immunization with currently available whole virus, split and subunit vaccines has several draw-backs: the effectiveness of these vaccines is dependent upon the continuously occurring antigenic drift or suddenly occurring antigenic shift of the membrane proteins of the influenza viruses, and they usually stimulate a short-life antibody response only. These facts have led to the recommendation to repeat vaccination annually. Moreover, published reports suggest, to various degrees, a limited efficacy of vaccines to protect aged subjects from influenza infection.

In principle, there are three ways to determine the efficacy of a given vaccine in human beings: (1) the experimental trial, (2) the field trial and (3) the immune response study.

1 In experimental trials, pathogenic effects are recorded in a vaccinated and unvaccinated group after challenge with a live pathogen. Experimental trials have been performed in healthy children and young adult volunteers with both wild or attenuated influenza strains (see Refs 5 and 6). It is evident that such a study design is impossible in aged populations.

2 In field trials, the challenge occurs by exposition of subjects to the natural field pathogen in the environment. Well-known general and methodological problems of field trials with naturally occurring influenza viruses include unpredictability of the time of its occurrence, antigenic differences between the vaccine strains and epidemic viruses, possible differences in the exposure rates of groups to be compared, and possible misdiagnoses of cases, if not laboratory-confirmed, because of the broad variety of other respiratory pathogens causing influenza-like symptoms. Special ethical problems arise in the aged. Since the benefits of influenza vaccines have been established in the sixties ${ }^{7}$, ethical reasons have limited the performance of field trials because of the risk to unvaccinated control subjects. As a consequence, most of the studies dealing with influenza-associated morbidity and mortality of vaccinated and unvaccinated aged populations are retrospective and/or suffer from a variety of serious methodological flaws.

Strassburg et al. ${ }^{8}$ reviewed 17 papers reporting influenza outbreaks in the aged and the effectiveness of influenza vaccination, during a period from 1967/68 to 
1982/83 and found important differences in study populations, vaccines, methodology and, as a consequence, levels of vaccine effectiveness. Despite these restrictions, they estimated average effectiveness rates for morbidity and mortality, respectively. While reduction of mortality in vaccinated subjects, as compared to unvaccinated controls, was calculated as being $67 \%$, reduction of morbidity was found to be much lower $(23 \%)$. However, experimental and field trials in young, healthy adults in various epidemiological settings, using various vaccines, have repeatedly revealed much higher rates for reduction of morbidity: $60-90 \%$ (for review see Ref. 9). Although comparison of such values should be made very carefully, it could appear that young subjects are better protected than the aged. Among the factors which may contribute to a possibly impaired protection rate in the aged, a direct, age-related impairment of the immunological response to vaccines is to be discussed. 3 The immune response study determines immunological changes after vaccination (mostly antibody production), which are thought to be associated with protection against the pathogen. The various methodological and ethical problems associated with field trials may be avoided. It is essential, of course, that the affirming variables for assessing the protective properties of the vaccine have been sufficiently established by experimental trials and field observations with regard to natural infection.

It is well known that serum IgG antibody against the viral haemagglutinin (HA) plays a major role in protection against influenza ${ }^{10,11}:$ in high concentrations, it provides resistance to acquisition of infection (total protection). In lower concentrations, it prevents or ameliorates disease after infection in a substantial number of cases (partial protection). Many authors define a quantitative protection threshold, i.e. a pre-exposition titre beyond which it is highly unlikely to acquire an infection or to develop illness. Thus, anti-HA serum antibody is a predictor of protection and a measure of vaccine efficacy, which makes immune response studies a good and practical alternative to field trials.

In the present paper, available studies dealing with the anti-HA serum antibody response in elderly subjects upon immunization with inactivated influenza vaccine, are reviewed in an attempt to obtain insight into the correlation between high age and sero-response. The collected information should stimulate further research which may lead to improved policies for the use of current vaccines in the elderly or to the development of new types of vaccines.

For reasons discussed later we did not apply quantitative methods for literature review to construct statistical summaries and comparisons of studies (meta-analysis ${ }^{12}$ ) but chose a traditional narrative approach (qualitative tally), though realizing its limitations ${ }^{13}$. However, we have attempted to obey the strict criteria for metaanalyses as established by Sachs et al. ${ }^{14}$.

\section{Materials and methods}

\section{Criteria for selection of papers and source of literature}

Age of study subjects. According to WHO practice ${ }^{15}$ the following terminology for chronological age is commonly used: $45-59$ years, middle age; $60-74$ years, elderly; 75-89 years, aged; 90 or more years, very old. However, chronological age is not always a good indicator of biological age, i.e. the decline in the physiological ability to react appropriately to environmental stimuli ${ }^{16}$. The process of biological aging is heterogeneous and occurs at different rates in individuals. For reasons of convenience and in spite of its arbitrary character, we used the terms 'elderly', 'aged' and 'very old' synonymously and selected papers dealing with persons predominantly above 60 years of age (aged groups), and control subjects younger than 60 years of age (young groups).

Biological predictor of protection, vaccines, study design and laboratory tests. As outlined above, the production of serum antibody against viral haemagglutinin was chosen as a predictor and measure of vaccine efficacy. The induction of this antibody is strongly induced, apart from natural infection, by immunization with inactivated (whole virus, split or subunit) vaccines, but less so by experimental live vaccines whose protective effects may predominantly be caused by stimulation of local immunity ${ }^{17}$. We did not include papers on these latter vaccines.

Any study design was accepted which included the drawing of two blood specimens, one before and a second after vaccination. Booster immunizations were not included. Any laboratory test detecting antibody against viral haemagglutinin was accepted. Tests dealing with antibodies directed against other viral proteins (neuraminidase, core) or measuring cellular immunity were not included, in view of their unknown association with protection.

Year of performance of the studies. The impure and highly reactogenic influenza vaccines used during the $40 \mathrm{~s}$, $50 \mathrm{~s}$ and $60 \mathrm{~s}$ were of variable potency. It did not seem appropriate for us to include this early vaccine period in our survey. At the end of the $60 \mathrm{~s}$, a new generation of highly purified, hardly reactogenic and potent wholevirus vaccines became available ${ }^{18.19}$, followed by split and subunit vaccines in the 70s. Coincidentally, in 1968 a new epidemiological situation occurred with the pandemic emergence of the influenza A-H3N2 subtype. Therefore, we only included studies performed from 1968 onwards.

Source of literature. English-language papers mentioned in the monthly 'Influenza Bibliography' of the Medical Research Council and the WHO World Influenza Centre, published by the Medical Research Council Library, National Institute for Medical Research, Mill Hill, London, UK, and the databases of the library computer system of the Medical Faculty of the Erasmus University Rotterdam were searched for the combination of the keywords 'influenza' and 'vaccination'. From these sources, papers were selected using the following criteria: age stratification of study subjects, type of vaccines, study design, laboratory tests, and year of performance of the study. The search was finished in December 1988.

\section{Serological response and statistical calculations}

Serological response may be expressed using one or more of the following parameters: (1) the mean fold increase (MFI), i.e. the difference between the logarithm 
of mean titres of post- and prevaccination sera, (2) the protection rate (PR), i.e. the proportion of subjects exceeding a given protection threshold after vaccination, (3) the response rate ( $R R$ ), i.e. the proportion of subjects who show an at least four-fold titre increase ${ }^{20,21}$ after vaccination.

The quantities corresponding with these parameters were either taken directly from the selected papers, or recalculated from their original data if given, or derived from appropriate tables or figures as presented in the original papers. For calculation of the protection rate, the protection threshold as given by the authors was used, or, if not available in the paper, a titre of $36-40^{11,22,23}$ when a standard microtitre haemagglutination inhibition technique had been performed. As outlined later, previous antibody against the vaccine component is a factor strongly influencing the production of new homologous antibody upon vaccination. While the protection rate is based on previously unprotected subjects, it has been attempted, for the two other parameters (MFI and RR), to retrospectively stratify the prevaccination state of the study groups as unprotected (including seronegative and low seropositive subjects) or protected subjects prior to vaccination, and to recalculate the quantities for unprotected subjects only. All recalculations are reported in the Tables.

For statistical analysis, the absolute differences between the seroresponse parameters of aged and young subjects were recalculated, and, for the protection and response rates, also the significance level $\left(\chi^{2}\right.$-test), and the type-2-error which is the probability of concluding that a difference does not exist, when it does in reality. By lack of original data, the significance level of differences between the MFI-values of aged and young groups could not be recalculated and was taken from the original papers if given.

\section{Criteria for the detection of selection biases in the literature}

Several factors may influence the production of antibodies after vaccination: health state of the study subjects, intake of drugs at the time of vaccination, and history of exposition to influenza antigens prior to vaccination. Ignoring these factors introduces substantial biases into a trial. Therefore, we examined the selected papers for addressing and correcting these factors.

Health state and use of drugs. Many acute and chronic illnesses (such as viral infections, malnutrition, renal and bone-marrow diseases etc.) and various drugs (such as analgesics, hormones, antimetabolites etc.) are known to impair the functions of the humoral immune system and should therefore lead to exclusion from immune response trials ${ }^{24}$.

Prevaccination antibody. Specific or cross-reacting antibody against a given influenza strain present in high concentrations before vaccination with this strain, may suppress or impair the production of new antibody. According to this 'law of initial values'25 or "negative feedback, 26 , the response to a given vaccine strain is inversely related to the specific prevaccination antibody titre. Possibly, already circulating antibody combines with the vaccine to mask its recognition by immunocompetent cells, or, alternatively, switches off new immunoglobulin synthesis by a more central mechanism ${ }^{26}$. Therefore, when performing vaccination trials, one should carefully describe the prevaccination state of the study populations and analyse the data for different prevaccination classes, or exclude persons with high prevaccination titres.

History of previous vaccinations. Vaccinations with heterologous influenza viruses in the past, even if they are no longer serologically detectable at the moment of the ungoing study (i.e. in the absence of prevaccination antibodies) may unpredictably influence the antibody production of the vaccine studied: sometimes a boostereffect is observed, sometimes a suppressive effect ${ }^{27}$. Subjects with previous vaccination should therefore be excluded.

\section{Results}

\section{Papers to be reviewed, and data on study design}

The sources revealed 1805 titles for the combination of the keywords 'influenza' and 'vaccination'. Only 20 papers met all our inclusion criteria. Many more papers dealt with influenza vaccination trials in the elderly but had not included control groups consisting of younger subjects. From the 20 selected papers, three ${ }^{28-30}$ were excluded because the anti-HA-IgG ELISA used did not discriminate between the different vaccine components.

Data of seven studies published in the period 1968-88 are presented in Table 1 in chronological order (years of performance of the trial, or, if this information had not been given, year of publication $)^{19,31-46}$. Most of the studies (10) were carried out in the United States of America. Only 10 studies (not shown) gave information about the seasonal relationship of the vaccination trial to natural influenza epidemics. Trials performed during or shortly after epidemics with considerable impact would make it difficult to decide whether titre rises were due to the vaccine or to the natural virus.

The period between collection of the pre- and postvaccination sera was usually $21-42$ days (not shown); two studies ${ }^{41,44}$ chose a shorter interval (14-15 days) which might be suboptimal, and two other papers ${ }^{39,43}$ did not give any information in that respect. As a technique for anti-HA-antibody determination, usually the haemagglutination inhibition test was performed, with the exception of Ref. 33 (neutralization test).

As in many studies bi- or trivalent vaccines had been used, the seroresponse to, in total, 30 vaccine components could be studied separately. When using various vaccine types $^{34}$ or various dosages ${ }^{19,42}$, the data on seroresponse were pooled, either by the authors themselves or by us, as there was a similar stratification among the study group of aged subjects and control groups. From 1967 onwards, the antigenic contents of commercial vaccines has been estimated by comparison with an international influenza A standard preparation and expressed in chicken cell agglutination (CCA) units (IU, international units). With the development of split and subunit vaccines, this technique became impracticable (see extremely high dosages in Refs. 38 and 39!) and was replaced by the single-radial immuno-diffusion test $(\mu \mathrm{g}$ haemagglutinin, HA) by the WHO in 1978 (for review see Ref. 47). 


\begin{tabular}{|c|c|c|c|c|c|}
\hline \multirow[b]{2}{*}{ Study ref. a.g } & \multirow[b]{2}{*}{ Year } & \multirow[b]{2}{*}{ Place $^{c}$} & \multicolumn{3}{|c|}{ Vaccines used } \\
\hline & & & Strain ${ }^{\sigma}$ & Type & $\operatorname{Dose}^{t}$ \\
\hline Gwaltney ${ }^{31}$ & 1968 & USA & A/Hong Kong/2/68 (H3N2) & WV & $400 \mathrm{CCA}$ \\
\hline Marine $^{32}$ & 1968 & USA & A/Aichi/2/68 (H3N2) & WV & $400 \mathrm{CCA}$ \\
\hline Fulk & 1968 & USA & A/Aichi/2/68 (H3N2) & WV & $400 \mathrm{CCA}$ \\
\hline Mostow ${ }^{19}$ & 1969 & USA & $\begin{array}{l}\text { A/Japan/170/62 (H2N2) } \\
\text { A/Aichi/2/68 (H3N2) }\end{array}$ & WV & $\begin{array}{l}300 / 3000 \mathrm{CCA} \\
300 / 3000 \mathrm{CCA}\end{array}$ \\
\hline Marine $^{35}$ & 1971 & USA & $\begin{array}{l}\text { A/Aichi/2/68 (H3N2) } \\
\text { A/Japan/305/57 (H2N2) }\end{array}$ & WV & $\begin{array}{l}1000 \mathrm{CCA} \\
1000 \mathrm{CCA}\end{array}$ \\
\hline Howells ${ }^{36}$ & 1971 & UK & $\begin{array}{l}\text { A/Hong Kong/2/68 (H3N2) } \\
\text { B/Victoria/70 }\end{array}$ & WV & $\begin{array}{l}400 \mathrm{CCA} \\
200 \mathrm{CCA}\end{array}$ \\
\hline Ruben $^{37}$ & 1972 & USA & A/Aichi/2/68 (H3N2) & SU & $700 \mathrm{CCA}$ \\
\hline McKenzie $e^{38}$ & 1973 & AUS & A/England/42/72 (H3N2) & SU & $16000 \mathrm{CCA}$ \\
\hline Feery ${ }^{39}$ & 1976 & AUS & $\begin{array}{l}\text { B/Hong Kong/8/73 } \\
\text { A/Port Chalmers } / 1 / 73 \text { (H3N2) }\end{array}$ & SU & $\begin{array}{r}8000 \mathrm{CCA} \\
16000 \mathrm{CCA}\end{array}$ \\
\hline Hannoun ${ }^{41}$ & 1977 & FR & A/New Jersey/8/76 (H1N1) & WV & $200 \mathrm{CCA}$ \\
\hline Beare $^{42}$ & 1978 & UK & A/New Jersey/8/76 (H1N1) & WV & $4-61 \mu \mathrm{g}$ \\
\hline Phair ${ }^{43}$ & 1978 & USA & $\begin{array}{l}\text { A/New Jersey/8/76 (H1N1) } \\
\text { A/Victoria/3/75 (H3N2) }\end{array}$ & SU & $\begin{array}{l}200 C C A \\
200 C C A\end{array}$ \\
\hline Feery ${ }^{44}$ & 1979 & AUS & $\begin{array}{l}\text { B/Hong Kong/8/73 } \\
\text { A/USSR/90/77 (H1N1) } \\
\text { A/Texas/1/77 (H3N2) }\end{array}$ & SU & $\begin{array}{l}250 \mathrm{CCA} \\
250 \mathrm{CCA} \\
250 \mathrm{CCA}\end{array}$ \\
\hline Gross $^{45}$ & 1984 & USA & $\begin{array}{l}\text { A/Philippines/2/82 (H3N2) } \\
\text { A/Chile/1/83 (H1N1) } \\
\text { B/USSR/100/83 }\end{array}$ & $\mathrm{SP}$ & $\begin{array}{l}15 \mu \mathrm{g} \\
15 \mu \mathrm{g} \\
15 \mu \mathrm{g}\end{array}$ \\
\hline Gross $^{46}$ & 1985 & USA & $\begin{array}{l}\text { A/Philippines/2/82 (H3N2) } \\
\text { A/Chile/1/83 (H1N1) } \\
\text { B/USSR/100/83 }\end{array}$ & SP & $\begin{array}{l}15 \mu \mathrm{g} \\
15 \mu \mathrm{g} \\
15 \mu \mathrm{g}\end{array}$ \\
\hline
\end{tabular}

${ }^{a}$ First author and reference number of paper

bYear of performance of the study or, if not given, year of publication of paper

${ }^{c}$ Country of performance of the study

${ }^{d}$ Abbreviated WHO-nomenclature of the virus strain used in the vaccine

'Vaccine type (WV, whole-virion; SU, subunit; NG, data not given)

'Vaccine doses (CCA, chicken cell agglutionation units; $\mu \mathrm{g}$, microgram $H A$ )

${ }^{g}$ Comment on study design. See appendix

\section{Data on populations studied}

Table 2 presents data to characterize the size, age range and prevaccination titres of the studied groups of aged and young subjects. The total numbers of groups varied from $10^{40}$ and $11^{33}$ up to 437 and $203^{34}$, for elderly and young groups, respectively. The age distribution for the elderly usually reached from 60-70 up to 101 years of age; the young groups consisted of subjects either of a particular narrow age cohort (children ${ }^{33}$, young adults $^{32,41}$ ) or a very broad age range ( $<65$ years). Three studies $^{31,37,45}$ did not report any age range. The first one characterized the aged group only by the term 'elderly population', the latter two gave high mean ages ( 80 years, 71-74 years). Another paper ${ }^{33}$ presented an age range also including persons younger than 60 years but the text suggested that those subjects had been few.

The elderly populations were usually recruited from old people's homes or geriatric institutions which was apparently reflected by the sex distribution: in all studies which gave data on that point, more female than male subjects had been included (not shown).

For 20 vaccine components, the prevaccination state of aged and young groups could be compared. For 10 strains, the elderly had a higher mean titre prior to vaccination; for four strains these values were equivocal, and in six instances the younger groups showed higher mean titres than the elderly.

\section{Serological results and statistical calculations}

Table 3 shows the results of the serological tests performed with pre- and postvaccination sera, and some basic statistical calculations.

MFI-values for aged and young groups were present in all but one paper; transformed to a logarithmic scale, the MFI varied in a wide range between 0.2-1.4 for aged and $0.2-1.6$ for young groups. For 15 of the 30 vaccine components, the authors themselves had given the significance level of the between-group differences for the MFI-values, which in none of these 15 cases was smaller than $5 \%$. The significance level for the remaining papers could not be recalculated by us because of missing raw data in the papers.

The protection and response rates were also heterogenous, reaching from 16 to $93 \%$ for aged, and from 21 to $100 \%$ for control groups. Between-group differences of protection rates could be calculated for 12 vaccine components, and of response rates for 16 vaccine components. Differences were only small for six protection rates and seven response rates and were not significant on a $5 \%$ level, but they had a high type-2-error (51-97\%). In these cases, interpretation is difficult as it is not possible to decide whether (1) there really were no differences between age groups or, (2) there were differences, however, the studies were too insensitive (too small sample sizes) to detect these. 
Table 2 Data on the study group composition of 17 selected papers

\begin{tabular}{|c|c|c|c|c|c|c|c|}
\hline \multirow[b]{2}{*}{$\begin{array}{l}\text { Study }{ }^{a} \\
\text { (Ref.) }\end{array}$} & \multirow[b]{2}{*}{ Strain } & \multicolumn{3}{|c|}{ Aged } & \multicolumn{3}{|c|}{ Young } \\
\hline & & No & $\begin{array}{l}\text { Age } \\
\text { range }\end{array}$ & $S 1^{b}$ & No & $\begin{array}{l}\text { Age } \\
\text { range }\end{array}$ & $\mathrm{S} 1^{b}$ \\
\hline 31 & A/HK/68 & 80 & $N G^{c}$ & 1.1 & 53 & NG & 0.8 \\
\hline 32 & $\mathrm{~A} / \mathrm{HK} / 68$ & 31 & $67-99$ & 1.3 & 23 & $23-25$ & 0.6 \\
\hline 33 & A/HK/68 & 16 & $50-87$ & NG & 11 & $6-14$ & NG \\
\hline \multirow[t]{2}{*}{19} & A/JAP/62 & 297 & $>65$ & 1.5 & 545 & $<65$ & 1.7 \\
\hline & $\mathrm{A} / \mathrm{HK} / 68$ & 293 & & 0.7 & 512 & & 0.7 \\
\hline 34 & A/HK/68 & 437 & NG & NG & 203 & NG & $N G$ \\
\hline \multirow[t]{2}{*}{35} & $\mathrm{~A} / \mathrm{HK} / 68$ & 40 & $80-101$ & NG & 70 & $6-31$ & NG \\
\hline & A/JAP/57 & 36 & NG & NG & 90 & $N G$ & NG \\
\hline \multirow[t]{2}{*}{36} & $\mathrm{~A} / \mathrm{HK} / 68 \mathrm{R}$ & 134 & $>60$ & 1.0 & 107 & $<65$ & 1.3 \\
\hline & $\mathrm{B} / \mathrm{VIC} / 70$ & & & 0.8 & & & 1.0 \\
\hline 37 & $\mathrm{~A} / \mathrm{HK} / 68$ & 57 & NG & 1.8 & 17 & NG & 0.9 \\
\hline 38 & A/ENG/72 & 69 & $60-79$ & 1.1 & 231 & $15-59$ & 1.0 \\
\hline \multirow[t]{2}{*}{39} & $\mathrm{~B} / \mathrm{HK} / 73$ & 61 & $68-93$ & 1.5 & 52 & $21-60$ & 1.3 \\
\hline & $\mathrm{A} / \mathrm{PC} / 73$ & & & 2.3 & & & 2.0 \\
\hline \multirow[t]{3}{*}{40} & $\mathrm{~B} / \mathrm{HK} / 73$ & 10 & $>60$ & 0.3 & 67 & $2-59$ & 0.6 \\
\hline & A/VIC/75 & & & 0.7 & & & 0.7 \\
\hline & $\mathrm{A} / \mathrm{NJ} / 76$ & & & 1.0 & & & 0.2 \\
\hline 41 & $\mathrm{~A} / \mathrm{NJ} / 76$ & 73 & $69-94$ & 1.4 & 57 & $18-20$ & 0.3 \\
\hline 42 & $\mathrm{~A} / \mathrm{NJ} / 76$ & 84 & $\geqslant 65$ & 1.9 & 372 & $13-65$ & $N G$ \\
\hline \multirow[t]{2}{*}{43} & A/NJ/76 & 72 & $60-95$ & 1.4 & 20 & $<45$ & NG \\
\hline & $\mathrm{A} / \mathrm{VIC} / 75$ & & & 1.2 & & & NG \\
\hline \multirow[t]{3}{*}{44} & $\mathrm{~B} / \mathrm{HK} / 73$ & 45 & $66-100$ & NG & 47 & $25-64$ & NG \\
\hline & A/USSR/77 & & & NG & & & NG \\
\hline & A/TEX/77 & & & NG & & & NG \\
\hline \multirow[t]{3}{*}{45} & A/PHIL/82 & 25 & NG & 1.3 & 21 & NG & 1.3 \\
\hline & A/CHIL/83 & & & 1.3 & & & 1.5 \\
\hline & B/USSR/83 & & & 1.4 & & & 1.5 \\
\hline \multirow[t]{3}{*}{46} & A/PHIL/82 & 56 & $>65$ & 1.3 & 45 & $<65$ & 1.3 \\
\hline & $\mathrm{A} / \mathrm{CHIL} / 83$ & & & 1.8 & & & 1.4 \\
\hline & B/USSR/83 & & & 1.7 & & & 1.5 \\
\hline
\end{tabular}

${ }^{a}$ Comment on group composition. See appendix

bPrevaccination mean titre of the entire group (decadic logarithm)

'Data not given (NG)

There were also significant between-group differences, 6 for the protection rate and 9 for the response rate, associated with a low $(<1-5 \%, 8$ cases $)$ or intermediate (16-42\%, 6 cases) type-2-error. For some vaccine components, a more favourable immune response for the young groups was revealed (significantly negative differences: 2 for PR and 8 for RR). However, also contrary results could be found (significantly positive differences: 4 for PR and 1 for RR).

Thus, a heterogeneous picture could be obtained, with more insignificant and significant results in favour of young subjects, which suggests a slight tendency.

An attempt to combine the pattern of MFI, PR and $R R$ per vaccine component to obtain a tendency per paper is shown in the last column of Table 3. Where the absolute differences of all given seroresponse measures had the same sign, this sign was used to indicate a tendency (either '-' for a better immune response of younger groups, or ' + ' for the alternative case). Where one of the given seroresponse measures was significant on the $5 \%$ level, the sign was doubled (either '- - ' or ' ++ '). Where the different seroresponse measures showed insignificant differences with different signs, this was expressed as ' 0 ' (no tendency detected).

Table 4 presents the 30 cases assessed in this way. For 16 cases, a 'significant' difference between the age groups was not detected (sum of cases indicated as ' - ', ' +' and ' 0 '). However, most of these ( 9 cases) suggest a slight tendency in favour of young age groups. This is supported by 10 cases with a clear tendency in favour of young groups, versus only four in favour of aged groups. The distribution of these results according to types or subtypes of virus strains does not reveal additional information for influenza A-H1N1, A-H2N2 and influenza $B$, because of the small number of cases per type/subtype. For influenza A-H3N2, results are contradictory, presenting both 3 'significant' cases in favour of young groups, and of aged groups, respectively.

\section{Selection biases and combinability of serological results}

Table 5 addresses three important criteria for group composition which should have been obeyed by the papers: absence of illness and drugs influencing the immune system, no previous influenza vaccinations, and stratification for or exclusion of protective prevaccination titres (or presentation of data in a way that this would be possible in retrospect). The documentation of these data was very incomplete in virtually all papers. In six cases it was stated, at least, that the study populations were clinically healthy. Two other papers reported that it dealt in part with 'chronically ill' subjects which did not lead to their exclusion. Another paper ${ }^{45}$ included children and young adults with cystic fibrosis as a control group. Only eight studies stated that the vaccinees, in the course of the study, had not used drugs influencing the immune system. Four papers reported that some of the participants had received other influenza vaccinations in previous years which, however, did not lead to 


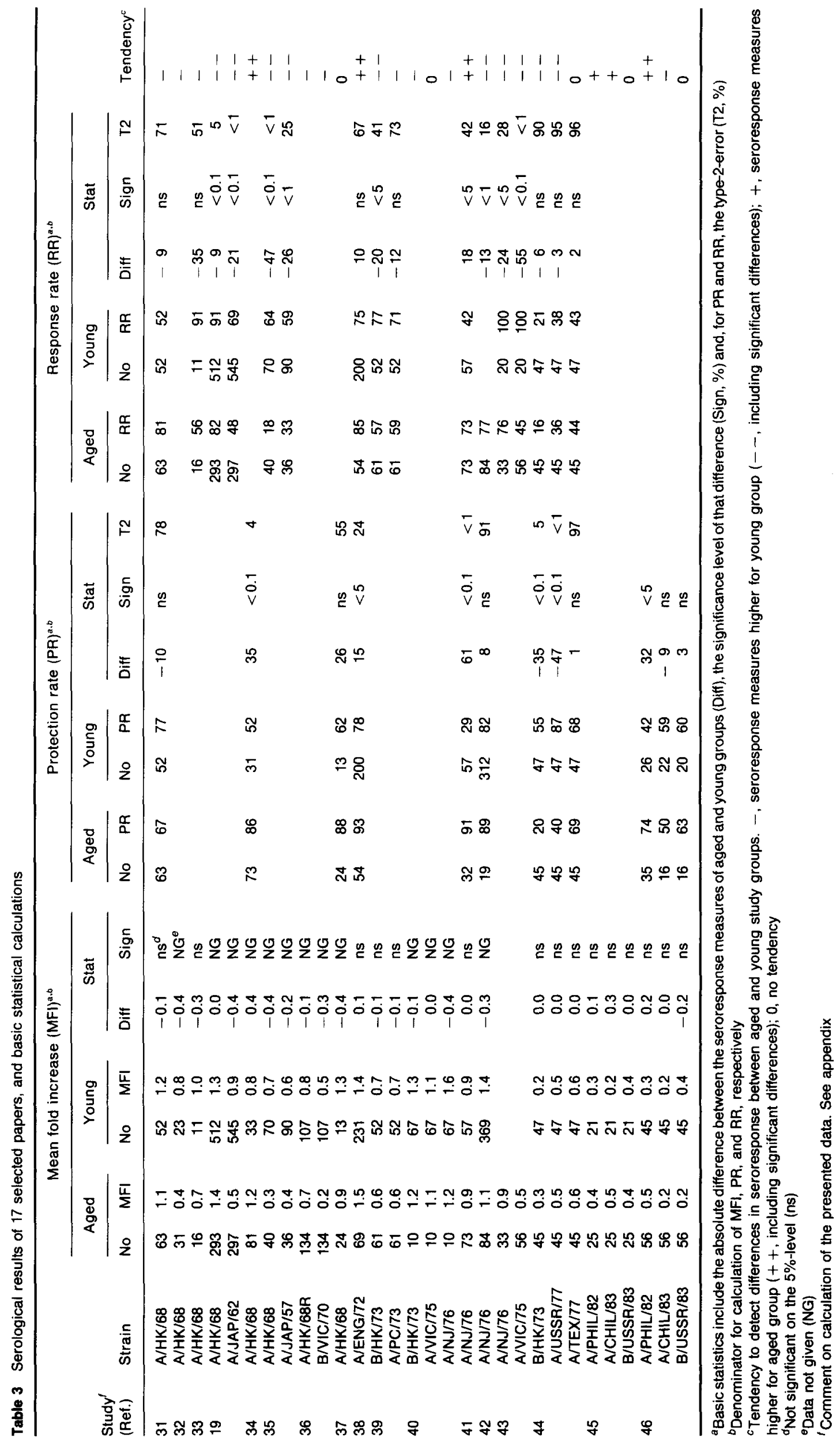


Table 4 Tendency of 17 selected papers to detect differences between aged and young study groups, subdivided for types and subtypes of 30 vaccine strains

\begin{tabular}{lccccc}
\hline & \multicolumn{5}{c}{ Tendency $^{a}$} \\
\cline { 2 - 6 } $\begin{array}{l}\text { Type/subtype } \\
\text { of vaccine strain }\end{array}$ & -- & - & 0 & + & ++ \\
\hline A-H1N1 & 3 & 2 & & 1 & 1 \\
A-H2N2 & 2 & & & & 3 \\
A-H3N2 & 3 & 5 & 3 & 1 & 3 \\
B & 2 & 2 & 2 & & 4 \\
Total $^{b}$ & 10 & 9 & 5 & 2 & 4
\end{tabular}

-, seroresponse variables higher for young group $(--$, including significant differences); + , seroresponse variables higher for aged group $(++$, including significant differences); 0 , no tendency

${ }^{b}$ Number of vaccine strains

exclusion of those subjects. Only one paper dealt expressis verbis with previously unvaccinated subjects. Thus, of the 17 controlled studies, none was free of any bias and 16 failed to address two or three criteria.

As the papers revealed a substantial heterogeneity in view of group composition and distribution of main biases which could not be corrected for, it did not appear to be possible to combine or pool the data on serological response (Table 3 ) in the sense of metaanalysis techniques.

\section{Discussion}

Influenza viruses are a major cause of mortality and serious morbidity in the elderly. Therefore, recommendations for the use of influenza vaccines have been given in several countries. Is vaccination really effective in this age-group? It is widely believed that, in the elderly, the ability to produce a sufficient amount of antibody after administration of bacterial and viral vaccines, is decreased, alongside with other age-related changes of the entire immune system. Early support for this impression came from the classical study of Sabin et al ${ }^{48}$ describing a lower immune response in aged people to Japanese B encephalitis virus vaccine, as compared to children. This paper has been cited frequently, but the observations of Sabin are based on 20 children and 20 aged individuals only, with a different history of previous expositions to the pathogen. Other support comes from field trials measuring the protection acquired by vaccination, against the naturally occurring pathogens (usually influenza viruses and Streptococcus pneumoniae), often suggesting a lower vaccine effectiveness in the elderly as compared to younger subjects. However, such studies usually struggle with methodological flaws ${ }^{8,49}$.

Only a few studies have been published which compare the seroresponse of different age groups to vaccines other than influenza. Results, as a working paper of the World Health Organization pointed out, are 'still incomplete and are often contradictory' ${ }^{\prime 5}$. Ammann et al. ${ }^{50}$ in testing a bivalent Streptococcus pneumoniae vaccine, found lower absolute antibody titres but a still sufficient protection rate, in a group of 66 elderly persons, as compared to 20 young adults. Musher et al. ${ }^{51}$ described 10 healthy young adults and five aged patients with chronic pulmonary disease after polyvalent Streptococcus pneumoniae vaccination and saw no significant differences in absolute postvaccination antibody titres, but a lower opsonizing activity, in the sera of the elderly. Other papers on Streptococcus pneumoniae vaccination not including young control groups, report satisfying antibody development in the aged ${ }^{52-57}$. Solomonova and Vizev ${ }^{58}$ detected a slightly delayed antibody production after vaccination with tetanus toxoid in aged subjects as compared to subjects aged 40-60 years. The difference, however, was not significant. In 70 non-dialysed elderly individuals, Denis et al. ${ }^{59}$ found a profoundly impaired seroresponse to hepatitis $B$ vaccine.

In an attempt to reveal, from the recent literature, the association between high age and the ability to produce specific antibodies after influenza vaccination, we could not detect a clear answer, but a tendency only: for 10 out of 30 single vaccine components, statistical calculations suggest a lower response of the elderly, as compared to younger control subjects. Another 9 cases support this tendency but show insignificant differences between age groups only. On the other hand, some contradictory results exist as well (elderly more favourable than young in 4 cases).

We showed that virtually all studies reviewed by us insufficiently controlled two major groups of biases: differences in health state and differences in previous exposure to influenza antigens. The general direction of these selection biases is such that failure to control these leads to an underestimation of the response of the elderly: in this age group, the prevalence of disease and drug use influencing the immune system, the chance of previous vaccinations, and the mean titres of prevaccination antibody, are generally higher. Neglecting these factors during the selection of the study subjects has introduced a considerable heterogeneity in the study groups of the papers reviewed and has also seriously affected the validity of the described results which may then give an idea about the overall condition of the group studied, but cannot lead to conclusions concerning fundamental age-related changes in the immune system. Although we saw a tendency supporting a decreased seroresponse in

Table 5 Selection biases present in 17 papers

\begin{tabular}{llll}
\hline & & \multicolumn{2}{c}{ Corrected for } \\
\cline { 2 - 4 } $\begin{array}{l}\text { Study } \\
\text { (Ref.) }\end{array}$ & $\begin{array}{l}\text { Health } \\
\text { state }\end{array}$ & $\begin{array}{l}\text { Vacc. } \\
\text { history }\end{array}$ & $\begin{array}{l}\text { Prevacc. }^{\text {state }} \\
\text { stal }\end{array}$ \\
\hline 31 & No & No & Yes \\
32 & No & No & No \\
33 & Yes & No & No \\
19 & No & No & No \\
34 & Yes & No & No \\
35 & No & No & No \\
36 & No & Yes & No \\
37 & No & No & Yes \\
38 & No & No & Yes \\
39 & No & No & No \\
40 & No & No & No \\
41 & No & No & No \\
42 & No & No & No \\
43 & Yes & No & Yes \\
44 & No & No & No \\
45 & No & No & No \\
46 & No & No & No \\
\hline
\end{tabular}

a Subjects excluded who had diseases or used drugs which may influence immune response

'Subjects excluded who had a history of previous influenza vaccinations 'Subjects excluded who had high (protecting) prevaccination titres 
Table 6 Selection and stratification criteria for influenza vaccination trials

\begin{tabular}{ll}
\hline Criterium & Controlled by \\
\hline Health state & $\begin{array}{l}\text { Senieur protocol } \\
\text { Eaccination history } \\
\text { Exclusion of subjects previously } \\
\text { immunized with any influenza vaccine } \\
\text { Exclusion of subjects with high/ } \\
\text { protective homologous serum antibody } \\
\text { prior to vaccination } \\
\text { Stratification according to priming } \\
\text { periods (see Table 7) }\end{array}$ \\
\hline
\end{tabular}

the elderly, the high prevalence of biases does not justify any generalization. The association between high age and responsiveness to influenza vaccines, if any, has not yet been established.

In this paper, we have used basic statistical methods analogous to another review on influenza vaccinology: Gross et al. ${ }^{60}$ reviewed 12 studies on the effects of cancer chemotherapy on the seroresponse to influenza vaccines and found that in eight papers a significantly lower antibody development in subjects on treatment, as compared to untreated control subjects, had been established. Moreover, they detected a high type-2-error in the remaining four studies which could not find any significant effects of chemotherapy which was probably due to insensitivity rather than to the absence of such differences. In contrast to the definite results of Gross et al., our results are more heterogeneous, even taking into account that many papers in our review had also a high type-2-error. The main distinction between the two reviews appears to be the fact that in the studies included by Gross et al., there was usually no major difference in age distribution, and, as a consequence, the two major groups of biases, differences in health state and in previous exposure to influenza antigens, were avoided.

Based on the considerations in the section Materials and methods of this review, we suggest admission criteria for future immune response studies in the elderly as described in Table 6. To define the health state of vaccinees, general criteria such as 'apparently healthy' or 'without overt diseases' are insufficient to exclude underlying disease, as pointed out by Ligthart et al. ${ }^{24}$. Therefore the strict 'Senieur Protocol: Admission criteria for immunogerontological studies in man' should be used as it includes clearly defined anamnestic and clinical information, laboratory data and drug interference. Subjects previously immunized with any hetero- or homologous influenza vaccines, and subjects who do not clearly remember, are to be excluded. Subjects with high/protective serum antibody prior to vaccination, specifically directed against the vaccine component, are to be excluded. The exclusion threshold may be dependent on the antibody detection method used.

In contrast to the three criteria mentioned above which can be controlled by an appropriate study design, the age-related exposition to different influenza $A$ subtypes is a factor which cannot be controlled. However, it strongly influences the immune response to vaccination even dozens of years later. Table 7 shows the natural occurrence of haemagglutinin subtypes from 1889 onwards, as has been established by seroepidemiological studies and, from 1933 onwards, by direct isolations in $\operatorname{man}^{61}$. In particular, the first influenza $A$ infection during
Table 7 Influenza A prevalence and priming eras

\begin{tabular}{llc}
\hline $\begin{array}{l}\text { Haemagglutinin } \\
\text { subtype }\end{array}$ & $\begin{array}{l}\text { Period of } \\
\text { prevalence }\end{array}$ & $\begin{array}{l}\text { Priming period } \\
\text { (birth years) }\end{array}$ \\
\hline $\mathrm{H} 2$ & $1889-1899$ & -1877 \\
$\mathrm{H} 3$ & $1890-1917^{\text {a }}$ & $1878-1905$ \\
$\mathrm{Hsw}$ & $1918-1925$ & $1906-1920$ \\
$?$ & $1926-1932$ & $1921-1927$ \\
$\mathrm{H} 0^{b}$ & $1933-1946$ & $1928-1939$ \\
$\mathrm{H} 1$ & $1947-1957$ & $1940-1949$ \\
$\mathrm{H} 2$ & $1958-1967$ & $1950-1961$ \\
$\mathrm{H} 3$ & $1968-^{-{ }^{\circ}}$ & $1962-$ \\
\hline
\end{tabular}

1908-1918, and 1978-co-circulation of $\mathrm{H3}$ and $\mathrm{H}^{\mathrm{B1}}$

${ }^{\circ}$ According to WHO-nomenclature of 1971. In the new nomenclature of 1980 . Hsw, $\mathrm{HO}$, and $\mathrm{H} 1$ are expressed as $\mathrm{H} 1$

lifetime ('priming') is of interest. It usually occurs at an age between 5 and 15 years and results in a potent immunological memory for the subtype to which the infective strain belongs. Individuals primed by a special subtype, react differently to later homologous or heterologous infections or vaccinations than those primed for another subtype (the doctrine of 'original antigenic $\sin ^{62}$ ). Therefore, age cohorts can be established according to primary infection. Table 7 presents the distribution of Marine and Thomas ${ }^{35}$, based on 687 persons of all ages in the United States in 1971 which has largely been confirmed by Masurel and Andre $(1978)^{63}$ who studied a population of 403 subjects in The Netherlands in 1977. The existence of age cohorts with differing natural exposure histories questions the validity of the general definition of 'aged' or 'elderly' as being 60 years of age or more, and suggests the necessity of a more differentiated age stratification according to the priming periods.

We believe that the use of strict selection and stratification criteria is mandatory if one wishes to study ageing per se. More knowledge of the latter is needed to develop appropriate strategies to protect elderly who do suffer from diseases and who have an increased risk of acquiring complications during or after influenza infection.

\section{Acknowledgements}

The authors are grateful to Dr G.J. Ligthart and Mrs N. Hamido for stimulating discussion, Mrs R.S. EngelsBakker for preparing the manuscript, and Mrs J.M.P. Den Ronden-De Jong for organizational assistance.

\section{References}

1 Alling, D.W., Blackwelder, W.C. and Stuart-Harris, Ch.H. A study of excess mortality during influenza epidemics in the United States, 1968-1976. Am. J. Epidemiol. 1981, 113, 30

2 Daver, C.C. and Serfling, R.E. Mortality from influenza. Am. Rev. Resp. Dis. 1961, 83, 15

3 Barker, W.H. and Mullooly, J.P. Impact of epidemic type A-influenza in a defined adult population. Am. J. Epidemiol. 1980, 112, 798

4 Hall, W.N., Goodman, R. A., Noble, G.R., Kendal, A.P. and Steece, R.S. An outbreak of influenza B in an elderly population. J. Infect. Dis. 1981, 144, 297

5 Delem, A. Serum SRH antibody level as a measure of the immunity against natural and artificial AVictoria/3/75 infections. Dev. Biol. Stand. 1977, 39, 391

6 Murphy, B.R., Markoff, L.J., Chanock, R.M., Douglas, R.G., Betts R.F., Cate, T.R. and Couch, R.B. An evaluation of influenza 
A/Victoria/3/75-ts-1[E] recombinant viruses for attenuation and immunogenicity in adult seronegative volunteers. Dev. Biol. Stand. $1977,39,47$

7 Stuart, W.H., Dull, H.B., Newton, L.H., McQueen, J.L. and Schiff, E.R Evaluation of monovalent influenza vaccine in a retirement community during the epidemic of 1965-1966. J. Am. Med. Ass. 1969 , 209, 232

8 Strassburg, M.A., Greenland, S., Sorvillo, F.J., Lieb, L.E. and Habel, L.A. Influenza in the elderly: report of an outbreak and a review of vaccine effectiveness reports. Vaccine 1986, 4, 38

9 Meiklejohn, G., Eickhoff, T.C. and Graves, P.I.J. Antigenic drift and efficacy of influenza virus vaccines, 1976-1977. J. Infect. Dis. 1978 138,618

10 Hobson, D., Curry, R.L. and Beare, A.S. Hemagglutination-inhibiting antibody titres as a measure of protection against influenza in man. Immunobiol. Stand. 1973, 20, 164

11 Potter, C.W. and Oxford, J.S. Determinants of immunity to influenza infection in man. Br. Med. Bull. 1979, 35, 69

12 Greenland, S. Quantitative methods in the review of epidemiologic literature. Epidemiol. Rev. 1987, 9, 1

13 Light, R.J. and Pillemer D.B. Summing Up: the Science of Reviewing Research. Harvard University Press, Cambridge MA, USA, 1984

14 Sacks, H.S., Berrier, J., Reitman, D., Ancona-Berk, V.A. and Chalmers, Th.C. Meta-analyses of randomized controlled trials. $N$. Engl. J. Med. 1987, 316, 450

15 World Health Organization. Immunization of the elderly. Report on a WHO Working Group. ICP/ESD 008, 1984

16 Makinodan, T. and Kay, M.M.B. Age influence on the immune system. In: Advances in Immunity, Vol. 29. Academic Press Inc., New York, 1980

17 Johnson, P.R., Feldman, S., Thompson, J.M., Mahoney, J.D. and Wright, P.F. Immunity to influenza $A$ virus infection in young children: a comparison of natural infection, live cold-adapted vaccine, and inactivated vaccine. J. Infect. Dis. 1986, 154, 121

18 Peck, F.B. Purified influenza virus vaccine: A study of viral reactivity and antigenicity. J. Am. Med. Ass. 1968, 206, 2277

19 Mostow, S.R., Schoenbaum, S.C., Dowdle, W.R., Coleman, M.T. and Kaye, H.S. Studies with inactivated influenza vaccines purified by zonal centrifugation. I. Adverse reactions and serological responses. Bull. Wid. Hith. Org. 1969, 41, 525

20 Dowdle, W.A., Kendal, A.P. and Noble, G.R. Influenza viruses. In: Diagnostic Procedures for Viral, Rickettsial and Chlamydial Infections. (Eds Lennette. E.H. and Schmidt. N.J.) 5th Edn., American Public Health Association, Washington, 1979, p. 585

21 Hawkes, R.A. General principles underlying laboratory diagnosis of viral infections. In: Diagnostic Procedures for Viral, Rickettsial and Chlamydial Infections. (Eds Lennette, E.H. and Schmidt, N.J.) 5th Edn., American Public Health Association, Washington, 1979 p. 29

22 Hobson, D., Curry, R.L., Beare, A.S. and Ward-Gardner, A. The role of serum haemagglutination-inhibiting antibody in protection against challenge infection with influenza A2 and B viruses. J. Hyg. 1972, 70, 767

23 Potter, C.W., Jennings, R., Nicholson, K. Tyrrell, D.A.J., and Dickinson, K.G. Immunity to attenuated influenza virus WRL 105 infection induced by heterologous, inactivated influenza $A$ virus vaccines. J. Hyg. 1977, 79, 321

24 Ligthart, G.J., Corberand, J.X., Fournier, C., Galanaud, P., Hijmans W., Kennes, B., $\theta t$ al.. Admission criteria for immunogerontological studies in man: the Senieur protocol. Mechanisms Ageing Develop. 1984, 28, 47

25 Voth, D.W., Feldman, H.A. and Steinschneider, A. Comparative responses of elderly persons to aqueous and depot influenza vaccines. Arch. Environ. Heatth 1966, 13, 576

26 Hobson, D., Baker, F.A. and Curry, R.L. Effects of influenza vaccines in stimulating antibody in volunteers with prior immunity. Lancet 1973, I, 155

27 Hoskins, T.W., Davies, J.R., Smith, A.J., Miller, C.L. and Allchin, A Assessment of inactivated influenza-A vaccine after three outbreaks of influenza A at Christ's hospital. Lancet 1979, i, 8106

28 Ershler, W.B., Moore, A.L., Roessner, K. and Ranges, G.E Interleukin-2 and aging: Decreased interleukin-2 production in healthy older people does not correlate with reduced helper cell numbers or antibody response to influenza vaccine and is not corrected in vitro by thymosin (alfa-1). $\mathrm{Imm}$. Pharmacology 1985 10. 11

29 Ershler, W.B., Moore, A.L. and Socinski, M.A. Influenza and aging Age-related changes and the effects of thymosin on the antibody response to influenza vaccine. J. Clin. Imm. 1984, 4, 445

30 Ershler, W.B., Moore, A.L. and Socinski, M.A. Specific antibody synthesis in vitro. III. Correlation of in vivo and in vitro antibody response to influenza immunization in young and old subjects. $J$. Clin. Lab. Imm. 1985, 16, 63

31 Gwaltney, J.M., Edmondson, W.P., Rothenberg, R. and White, P.W. A comparison of subcutaneous, nasal and combined influenza vaccination. I. Antigenicity. Am. J. Epidemiol. 1971, 93, 472

32 Marine, W.M., Workman, W.M. and Webster, R.G. Immunological interrelationships of Hong Kong, Asian and Equi-2 influenza viruses in man. Bull. Wid. Hith. Org. 1969, 41, 475

33 Fulk, R.V., Fedson, D.S., Huber, M.A., Fitzpatrick, J.R., Howar, B.F. and Kasel, J.A. Antibody responses in children and elderly persons following local or parenteral administration of an inactivated influenza virus vaccine, A2/Hong Kong/68 variant. J. $/ \mathrm{mm}$. 1969, 103,1102

34 Cromwell, H.A., Brandon, F.B., McLean, I.W. and Sadusk, J.F. Influenza immunization: A new vaccine. J. Am. Med. Assoc. 1969 210, 1438

35 Marine, W.M. and Thomas, J.E. Age-related response to 1000 CCA units zonally purified, inactivated influenza vaccines in volunteers in the U.S.A. Postgrad. Med. J. 1973, 49, 164

36 Howells, C.H.L., Vesselinova-Jenkins, C.K., Evans, A.D. and James, $\mathrm{J}$. Influenza vaccination and mortality from broncho-pneumonia in the elderly. Lancet 1975, I, 381

37 Ruben, F.L. Effectiveness of current killed influenza vaccines against influenza A/England/42/72. J. Infect. Dis. 1973, 127, 576

38 Mackenzie, J.S. Influenza subunit vaccine: Antibody responses to one and two doses of vaccine and length of response, with particular reference to the elderly. Br. Med. J. 1977, 1, 200

39 Feery, B.J., Evered, M.G. and Morrison, E.I. Antibody responses to influenza virus subunit vaccine in the aged. Med. J. Aust. 1976, 1, 540

40 Sarateanu, D., Ehrengut, W. and Pressler, K. Serological response to an adsorbed killed trivalent influenza vaccine (including $\mathrm{A} / \mathrm{New}$ Jersey/8/76 antigen). Dev. Biol. Stand. 1977, 39, 235

41 Hannoun, C., Barme, M., Sérié, C., Beck, H., Aquino, J. and Thibon $M$. Antibody response to anti-A/New Jersey/76 vaccines. Dev. Biol. Stand. 1977, 39, 249

42 The Pandemic Working Group of the Medical Research Council (United Kingdom) Committee on Influenza and Other Respiratory Virus Vaccines. Antibody responses and reactogenicity of graded doses of inactivated influenza A/New Jersey/76 whole-virus vaccine in humans. J. Infect. Dis. 1977, 136, $\$ 475$

43 Phair, J., Kauffman, C. A., Bjornson, A., Adams, L. and Linnemann, $C$. Failure to respond to influenza vaccine in the aged: correlation with B-cell number and function. J. Lab. Clin. Med. 1978, 92, 822

44 Feery, B. J., Gallichio, H. A., Rodda, S.J. and Hampson, A.W. Antibody responses to influenza vaccines containing A/USSR/90/77. Austr. J. Biol. Sci. 1979, 57, 335

45 Gross, P. A, Quinnan, G. V., Weksler, M.E., Gaerlan, P.F. and Denning, C.R. Immunization of elderly people with high doses of influenza vaccine. J. Am. Geriat. Soc. 1988, 36, 209

46 Gross, P.A., Weksler, M.E., Quinnan Jr., G.V., Douglas Jr., R.G. Gaerlan, P.F. and Denning, C.R. Immunization of elderly people with two doses of influenza vaccine. J. Clin. Microbiol. 1987, 25, 1763

47 Majer, M., Kindt, H. and Mettier, B. Fuenf Jahre Erfahrung mit einem Influenza-Subunitimpfstoff. Imm. Infect. 1981, 9, 232

48 Sabin, A.B., Ginder, D.R., Matumoto, M. and Schlesinger, R.W. Serological response of Japanese children and old people to Japanese $B$ encephalitis mouse brain vaccine. Proc. Sox. Exp. Biol Med. 1947, 65135

49 Schwartz, J.S. Pneumococcal vaccine: Clinical efficacy and effectiveness. Ann. Int. Med. 1982, 96, 208

50 Ammann, A.J., Schiffmann, G. Austrian, R. The antibody responses to pneumococcal capsular polysaccharides in aged individuals Proc. Soc. Exp. Biol. Med. 1980, 164, 312

51 Musher, D.M., Chapman, A.J., Goree, A., Jonsson, S., Briles, D. and Baughn, R.E. Natural and vaccine-related immunity to Streptococcus pneumoniae. J Infect. Dis. 1986, 154, 245

52 Kaufman, P. Pneumonia in old age. Active immunization against pneumonia with pneomococcus polysaccharide; results of a six year study. Arch. Int. Med. 1947, 79, 518

53 Schouten, J. Immune response to a 14-valent pneumococcal polysaccharide vaccine in the elderly. Pharmatherapeutica 1981, 3,

54 Hilleman, MR Carison, A J Mclean, A A Vella, P. P Weibel R.E. and Woodhour, A.F. Streptococcus pneumoniae polysaccharide: vaccine age and dose responses, safety, persistence of antibody, revaccination, and simultaneous administration of pneumococcal and influenza vaccines. Rev. Inf. Dis. 1981, 3, S1

55 Bentley, D.W. Pneumococcal vaccine in the institutionalized elderly: Review of past and recent studies. Rev. Infect. Dis. 1981, 3, S6

56 Landesman, S.H., Smith, P.M. and Schiffman, G. Pneumococcal 
vaccine in elderly patients with COPD. Chest 1983, 84, 433

57 Roghmann, K.J., Tabloski, P. A., Bentley, D.W. and Schiffman, G. Immune response of elderly adults to Pneumococcus: Variation by age, sex, and functional impairment. J. Geront. 1987, 42, 265

58 Solomonova, K., Vizev, S. Immunological reactivity of senescent and old people actively immunized with tetanus toxoid. $Z$. Immun.Forsch. 1973, 146, 81

59 Denis, F., Mounier, M., Hessel, L., Michel, J.P., Gualde, N., Dubois, F., Barin, F. and Goudeau, A. Hepatitis-B vaccination in the elderly. $J$ Infect. Dis. 1984, 149, 1019

60 Gross, P.A., Gould, A.L. and Brown, A.E. Effect of cancer chemotherapy on the immune response to influenza virus vaccine review of published studies. Rev. Infect. Dis. 1985, 7, 613

61 Masurel, N. and Heijtink, R.A. Recycling of H1N1 influenza A virus in man - a haemagglutinin antibody study. J. Hyg. 1983, 90, 397

62 Francis, T., Davenport, F.M. and Hennessy, A.V. A serologic recapitulization of human infection with different strains of influenza virus. Trans. Assoc. Am. Phys. 1953, 66, 231

63 Masurel, N. and André, F.E. Antibody response against current H1N1 influenza virus after vaccination with last season's trivalent vaccine. Lancet 1978, I, 144

64 Howells, C.H.L., Evans, A.D. and Vesselinova-Jenkins, C. Effect of two doses of influenza vaccine in stimulating antibody in volunteers. Lancet 1973, i, 1436

65 Ruben, F.L., Johnston, F. and Streiff, E.J. Influenza in a partially immunized aged population. J. Am. Med. Assoc. 1974, 230, 863

\section{Appendix}

\section{Comments}

Gwaltney et al. $^{31}$.

Four study groups were described: 'spray group', 'control group', 'combined group' and 'gun group'. Only the latter two were included here. Data for the neutralization test were not included. PR and RR were read from Figure 1.

Marine et al. ${ }^{32}$ :

MFI-values were read from Figures 2 and 3.

Fulk et al. $^{33}$ :

Groups vaccinated either locally, or parenterally, were described. Only the latter ones were included here. $\log _{2}$ values in Table $I$ of the original papers were transformed to $\log _{10}$ values.

Mostow et al. ${ }^{19}$ :

Data for 'prison group (GSP)' and 'school group (OCS)' were pooled to form the young group here. Table 5, which presented the seroresponse, gave only percentages without the appropriate denominators. These had partly to be taken from Table 1 of the original paper, partly to be calculated using a short information, in brackets, in Materials and methods.

Marine and Thomas ${ }^{35}$ :

Groups 1 and 2 (birth date 1940-1965), but not group 3 (birth date 1892-1939) of Table 4 of the original paper were pooled to form the young group here.

Howells et al. ${ }^{36}$ :

Vaccination trials from 1971 to 1973 were described. Only data for 1971 were included here. The data for the young group came from a different paper ${ }^{64}$. Only seroconversion from negative prevaccination titres to postvaccination titres greater than 10 , and mean postvaccination titres were given.
Ruben $^{37}$.

Also Ref. 65 was reviewed (identical experiment). In the original paper, bivalent vaccine (A/Aichi/2/68 and $\mathrm{B} / \mathrm{Mass} / 1 / 71$ ) was described, but no data were presented for the latter strain. It should be mentioned that vaccination of the control group had been performed one year earlier and with a higher vaccine dose.

Mackenzie $^{38}$ :

A bivalent vaccine (A/England/42/72 and $B /$ Roma/1/67) was used, but no data were presented for the latter strain. In Tables $I I I$ and $I V$ of the original paper, primary and booster vaccination were combined while, in the first two Tables, the effects of both injections could be studied separately. Moreover, the authors included only seroresponders in Tables $I I I$ and $I V$ of the original paper.

Sarateanu et al..$^{40}$ :

Age groups 'I-III' of the original paper were pooled to form the young group.

Hobson et al. ${ }^{26}$ :

A trivalent vaccine (A/New Jersey/8/76, A/Victoria/75 and $\mathrm{B} /$ Hong Kong/2/8/73) was used, but no data were presented for the latter two strains. Calculation of $51: 0.5 \log _{5}$ for negative sera. In forming the young group, only data of young persons receiving the identical doses as aged subjects (200 CCA), were used.

The Pandemic Working Group etc. ${ }^{42}$ :

The numbers and percentages of Tables 2, 3 and 4 of the original paper did not fit well. For example, in Table 3, $11 \%$ of the subjects $>65$ years had a prevaccination titre greater than 10 but in Table 4, 13\%.

Feery et $a .^{44}$ :

The young group was formed only by the group named 'adults' in the original paper; another group named 'young adults' was excluded here because these subjects had received a booster immunization.

Gross et al. ${ }^{45}$ :

Two more aged groups were vaccinated with higher doses of the trivalent split vaccine, and three more groups with different doses of a whole virus vaccine. The total number of persons was 148, divided into six groups. The mean ages of the groups ranged between 71 and 74 years. The control groups were children and young adults with cystic fibrosis. For our calculations, we used the data of Table 2 of the original paper. Protection rates could not be recalculated as numbers of protected subjects before vaccination were not given.

Gross et al. ${ }^{46}$ :

Two more aged groups ( $\mathrm{C}$ and $\mathrm{D}$ in Table 1 of the original paper) were vaccinated with whole virus vaccine. These data were excluded because of the lack of a young reference group. Data on booster vaccination in the elderly were excluded. The numbers of aged groups vaccinated with split vaccine in Tables 1 and $2(n=56)$, and Table $3(n=25)$ of the original paper differ. For our calculations, we used the data of Tables 1 and 2 of the original paper. 